JOURNAL OF THE

AMERICAN MATHEMATICAL SOCIETY

Volume 12, Number 4, Pages 1091-1102

S 0894-0347(99)00314-8

Article electronically published on June 15, 1999

\title{
ON THE STABLE RANK OF SIMPLE C*-ALGEBRAS
}

\author{
JESPER VILLADSEN
}

\section{INTRODUCTION}

In [16] M. A. Rieffel introduced the notion of stable rank for $\mathrm{C}^{*}$-algebras. For $A$ a unital $\mathrm{C}^{*}$-algebra the stable rank, denoted by $\operatorname{sr}(A)$, is the least integer $n$ such that the set of $n$-tuples over $A$ which generate $A$ as a left ideal is dense in $A^{n}$ if no such $n$ exists we set $\operatorname{sr}(A)=\infty$. For unital $\mathrm{C}^{*}$-algebras the stable rank was shown to coincide with the Bass stable rank, which is defined for rings; see [12]. The stable rank can also be defined for nonunital $\mathrm{C}^{*}$-algebras, but we shall only consider the unital case.

Stable rank is related to nonstable K-theory: In [16, Theorem 10.12] and [3, Corollary 7.14] it was shown that if $A$ is a simple unital $\mathrm{C}^{*}$-algebra of stable rank one, then the natural map $\mathrm{U}(A) / \mathrm{U}(A)_{0} \rightarrow \mathrm{K}_{1}(A)$ is an isomorphism. See also [17], $[2]$ and $[18]$.

It is a natural question which values occur as the stable rank of simple $\mathrm{C}^{*}$ algebras. Suppose that $A$ is simple and infinite. Then, by [7, Proposition 1.5], $A$ contains two isometries with orthogonal ranges and so, by [16, Proposition 6.5], $\operatorname{sr}(A)=\infty$. In the case of finite, simple $\mathrm{C}^{*}$-algebras the following is known: Whenever $A$ is simple and stably finite and $B$ is a UHF-algebra, the tensor product $A \otimes B$ has stable rank one; see [19]. This is also the case for every simple approximately homogeneous $(\mathrm{AH})$ algebra with slow dimension growth; see [4] and [8]. So, in short, $\mathrm{sr}=\infty$ for every infinite simple $\mathrm{C}^{*}$-algebra and $\mathrm{sr}=1$ for various classes of finite simple $\mathrm{C}^{*}$-algebras.

In this paper we answer the question above by showing that every $n \in \mathbb{N} \cup\{\infty\}$ is the stable rank of some simple, finite $\mathrm{C}^{*}$-algebra. More precisely, it is shown that for every $n=2,3,4, \ldots, \infty$ there exists a simple, separable and unital AH-algebra $A$ of stable rank $n$. In particular, the group of invertible elements in $A$ is not dense. And, like the examples in [20], $A$ does not have slow dimension growth and is not approximately divisible; see [5].

I thank Marius Dădărlat for suggesting that I study AH-algebras with connecting homomorphisms as described in the following section, and Juliana Erlijman for drawing my attention to some of the powerful tools of differential topology, and I thank George Elliott for my stay at The Fields Institute, where this paper was written.

Received by the editors May 18, 1998 and, in revised form, March 23, 1999.

1991 Mathematics Subject Classification. Primary 46L05; Secondary 46L80, 46M20.

Key words and phrases. Stable rank, simple $\mathrm{C}^{*}$-algebra, nonstable K-theory, real rank.

(C)1999 American Mathematical Society 


\section{DiAgONAL $*$-HOMOMORPHISMS}

Let $\mathcal{K}$ denote the $\mathrm{C}^{*}$-algebra of compact operators on an infinite-dimensional, separable Hilbert space $\mathcal{H}$. Suppose that $X$ is a compact space and $p$ a projection in $C(X) \otimes \mathcal{K}$ of constant rank. Then the image of $p(x)(x \in X)$ is a complex subspace of $\mathcal{H}$ and in this way $p$ gives rise to a complex vector bundle over $X$. Note that two such vector bundles are isomorphic if and only if the corresponding projections are equivalent in the sense of Murray and von Neumann.

$\mathrm{A} *$-homomorphism $\phi: C(X) \otimes \mathcal{K} \rightarrow C(Y) \otimes \mathcal{K}$ is called diagonal if there exist $k \in \mathbb{N}$ and maps $\lambda_{1}, \ldots, \lambda_{k}: Y \rightarrow X$ and mutually orthogonal projections $p_{1}, \ldots, p_{k}$ in $C(Y) \otimes \mathcal{K}$ such that $\phi=\left(\operatorname{id}_{C(Y)} \otimes \alpha\right) \circ\left(\tilde{\phi} \otimes \operatorname{id}_{\mathcal{K}}\right)$ where

$$
\tilde{\phi}: C(X) \rightarrow C(Y) \otimes \mathcal{K}, \quad f \mapsto \sum_{i=1}^{k}\left(f \circ \lambda_{i}\right) p_{i}
$$

and $\alpha$ is some isomorphism $\mathcal{K} \otimes \mathcal{K} \rightarrow \mathcal{K}$. In this case we say that $\phi$ comes from the tuple $\left(\lambda_{i}, p_{i}\right)_{i=1}^{k}$.

The composition of two diagonal $*$-homomorphisms is again diagonal. Indeed, if $\phi: A \rightarrow B$ comes from $\left(\lambda_{i}, p_{i}\right)_{i}$ and $\psi: B \rightarrow C$ comes from $\left(\mu_{j}, q_{j}\right)_{j}$, then a small computation shows that $\psi \circ \phi$ comes from $\left(\lambda_{i} \circ \mu_{j},\left(p_{i} \circ \mu_{j}\right) q_{j}\right)_{i, j}$.

Thinking of projections in a homogeneous algebra as complex vector bundles and adopting the corresponding notation — as we will do throughout this paper - one can easily verify that if $\phi$ is a diagonal $*$-homomorphism which comes from $\left(\lambda_{i}, \zeta_{i}\right)_{i}$ and $\xi$ is a projection, then $\phi(\xi) \cong \bigoplus_{i} \lambda_{i}^{*}(\xi) \otimes \zeta_{i}$.

\section{The COnstruction}

For $B$ a $C^{*}$-algebra with unit, we denote by $\operatorname{Lg}_{k}(B)$ the set of $k$-tuples in $B$ which generate $B$ as a left ideal:

$$
\begin{aligned}
\operatorname{Lg}_{k}(B) & =\left\{\left(b_{1}, \ldots, b_{k}\right) \in B^{k} \mid B b_{1}+\cdots+B b_{k}=B\right\} \\
& =\left\{\left(b_{1}, \ldots, b_{k}\right) \in B^{k} \mid \exists c_{1}, \ldots, c_{k} \in B: \sum_{i=1}^{k} c_{i} b_{i}=1\right\} .
\end{aligned}
$$

The stable rank of $B$ is then, by definition, the least integer $k$ such that $\operatorname{Lg}_{k}(B)$ is dense in $B^{k}$.

Let $n \in \mathbb{N}$ be fixed. We will construct a simple, unital AH-algebra $A$ such that $\operatorname{sr}(A)=n+1$; in the last section we show how to modify the construction to get infinite stable rank. First, let $\sigma(k)=k k$ ! for $k \in \mathbb{N}$ and put

$$
X_{i}=\mathbb{D}^{n} \times \mathbb{C P}^{n \sigma(1)} \times \mathbb{C P}^{n \sigma(2)} \times \cdots \times \mathbb{C P}^{n \sigma(i)},
$$

where $\mathbb{D}$ denotes the closed unit disc in the complex plane, $\mathbb{D}^{n}$ the $n$-fold Cartesian product and $\mathbb{C P}^{k}$ is complex projective $k$-space. Then $X_{0}=\mathbb{D}^{n}$ and $X_{i+1}=$ $X_{i} \times \mathbb{C P}^{n \sigma(i+1)}$ and we let

$$
\pi_{i+1}^{1}: X_{i+1} \rightarrow X_{i}, \quad \pi_{i+1}^{2}: X_{i+1} \rightarrow \mathbb{C P}^{n \sigma(i+1)}
$$

denote the two coordinate projections. Let $\gamma_{k}$ be the universal line bundle over $\mathbb{C P}^{k}$ and put $\zeta_{i}=\pi_{i}^{2 *}\left(\gamma_{n \sigma(i)}\right)$. Choose a dense sequence $\left(z_{i}^{\ell}\right)_{\ell=1}^{\infty}$ in $X_{i}$ and choose for each $j=1,2, \ldots, i+1$ a point $y_{i}^{j} \in X_{i}$ such that $y_{i}^{i+1}=z_{i}^{1}, y_{i}^{i}=z_{i}^{2}$ and $\pi_{j+1}^{1} \circ \pi_{j}^{1} \circ \ldots \circ \pi_{i}^{1}\left(y_{i}^{j}\right)=z_{j}^{i-j+2}$ for $1 \leq j \leq i-1$. Let $\tilde{\phi}_{i}: C\left(X_{i}\right) \otimes \mathcal{K} \rightarrow C\left(X_{i+1}\right) \otimes \mathcal{K}$ be a diagonal $*$-homomorphism which comes from $\left(\pi_{i+1}^{1}, \theta_{1}\right) \cup\left(y_{i}^{j}, \zeta_{i+1}\right)_{j=1}^{i+1}$ - as 
described above $\left(\theta_{1}\right.$ denotes a trivial line bundle). Now, let $p_{0} \in C\left(X_{0}\right) \otimes \mathcal{K}$ be a projection which corresponds to a trivial line bundle and put $p_{i}=\tilde{\phi}_{i 0}\left(p_{0}\right), i \in \mathbb{N}$. Let $A_{i}=\left(C\left(X_{i}\right) \otimes \mathcal{K}\right)_{p_{i}}$, and let $\phi_{i}: A_{i} \rightarrow A_{i+1}$ be the restriction of $\tilde{\phi}_{i}$. Then $\phi_{i}$ is unital. Let $A$ denote the $\mathrm{C}^{*}$-algebra $\underset{\lim }{\longrightarrow}\left(A_{i}, \phi_{i}\right)$. Then $A$ is a simple, unital AH-algebra.

That $A$ is simple follows essentially from [8], but for the convenience of the reader a proof is given here: Suppose that $I \neq 0$ is a closed, two-sided ideal in $A$. Then the union of $I \cap \phi_{\infty i}\left(A_{i}\right)$, over all $i$, is dense in $I$ and so there exist $i$ and a nonzero $a \in A_{i}$ such that $\phi_{\infty i}(a) \in I$. Since $\left(z_{i}^{\ell}\right)$ is dense in $X_{i}$ there exists $\ell \in \mathbb{N}$ such that $a\left(z_{i}^{\ell}\right) \neq 0$. Now, $z_{i}^{\ell}$ is among the point evaluations in the composition $\phi_{\ell+i-1 i}=\phi_{\ell+i-2} \circ \ldots \circ \phi_{i}$. Consequently, $\phi_{\ell+i-1 i}(a)$ is everywhere nonzero. Clearly, the preimage $\phi_{\infty \ell+i-1}^{-1}(I)=: J$ is a closed two-sided ideal of $A_{\ell+i-1}$, so there exists a closed subset $F \subseteq X_{\ell+i-1}$, such that $J$ consists exactly of those elements which, when viewed as matrix-valued functions on the spectrum, vanish on $F$. Since $\phi_{\ell+i-1 i}(a)$ is everywhere nonzero and belongs to $J$ we see that $F$ is empty, so that $J=A_{i}$. Since the $\phi_{i}$ 's are all unital, $I=A$. This shows that $A$ is simple.

At this point we need some notation and basic facts about the Euler class of complex vector bundles and the formation of the Cartesian product.

Consider the map $\times: \mathrm{H}^{*}(A) \otimes \mathrm{H}^{*}(B) \rightarrow \mathrm{H}^{*}(A \times B)$ given by $\times(a \otimes b)=\rho_{1}^{*}(a) \rho_{2}^{*}(b)$ (cup multiplication) where $\rho_{1}$ and $\rho_{2}$ are the coordinate projections from $A \times B$ to $A$ and $B$ respectively. For simple tensors we write $\times(a \otimes b)=a \times b$. By the Künneth formula $\times$ is injective when, for instance, $A$ and $B$ are finite $C W$-spaces; see [9]. Now, suppose that this is the case and that $\xi$ and $\zeta$ are complex vector bundles over $A$ and $B$ respectively. The Cartesian product $\xi \times \zeta$ is isomorphic to $\rho_{1}^{*}(\xi) \oplus \rho_{2}^{*}(\zeta)$ so the Euler class $\mathrm{e}(\xi \times \zeta)$ equals $\mathrm{e}(\xi) \times \mathrm{e}(\zeta)$. Hence, if neither $\mathrm{e}(\xi)$ nor $\mathrm{e}(\zeta)$ is zero or a torsion element, then the same holds for $\mathrm{e}(\xi \times \zeta)$; see [14].

From the definition of $\phi_{i}$ we see that $\operatorname{rank}\left(p_{i+1}\right)=(i+2) \operatorname{rank}\left(p_{i}\right)$ and since $\operatorname{rank}\left(p_{0}\right)=1$ we find that $\operatorname{rank}\left(p_{i}\right)=(i+1)$ !. Let $\xi_{i}$ denote the complex vector bundle over $X_{i}$ which corresponds to $p_{i}$. Using the above expression for $\operatorname{rank}\left(p_{i}\right)$ we see from the definition of $\phi_{i}$ that $\xi_{i+1} \cong \pi_{i+1}^{1 *}\left(\xi_{i}\right) \oplus \sigma(i+1) \zeta_{i+1}$, where $k \zeta$ denotes the $k$-fold direct sum of $\zeta$ by itself. It follows that

$$
\xi_{i} \cong \theta_{1} \times \sigma(1) \gamma_{n \sigma(1)} \times \cdots \times \sigma(i) \gamma_{n \sigma(i)}
$$

and therefore

$$
\mathrm{e}\left(\xi_{i} \ominus \theta_{1}\right)^{n}=\mathrm{e}\left(\gamma_{n \sigma(1)}\right)^{n \sigma(1)} \times \cdots \times \mathrm{e}\left(\gamma_{n \sigma(i)}\right)^{n \sigma(i)} .
$$

Using the fact that $\mathrm{e}\left(\gamma_{k}\right)^{k}$ in $\mathrm{H}^{2 k}\left(\mathbb{C P}^{k}\right)$ is nonzero it follows from the Künneth formula, as described above, that $\mathrm{e}\left(\xi_{i} \ominus \theta_{1}\right)^{n}$ is nonzero.

Before we can prove that $\operatorname{sr}(A)=n+1$, in Theorem 8 , we need some preparations. But before that, let me list the properties of the construction which we will need: First of all $A_{0}=C\left(\mathbb{D}^{n}\right)$ and the spectrum of $A_{i}$ is of the form $\mathbb{D}^{n} \times M_{i}$, where $M_{i}$ is a compact, oriented, smooth manifold. Secondly, there exists, for each $i$, a trivial projection $q_{i}$ in $A_{i}$ of rank 1 whose orthogonal complement corresponds to a bundle for which the $n$ 'th power of the Euler class is nonzero, and

$$
q_{i} \phi_{i 0}(f) q_{i}=\left(f \circ \pi_{i}\right) q_{i}
$$

for $f \in A_{0}$, where $\pi_{i}$ is projection of $\mathbb{D}^{n} \times M_{i}$ onto $\mathbb{D}^{n}$. 
Let $a_{j} \in A_{0}=C\left(\mathbb{D}^{n}\right)$ be projection onto the $j$ 'th coordinate. The elements $a_{1}, \ldots, a_{n}$ will play an important rôle. We will namely show that the distance from $\left(\phi_{i 1}\left(a_{1}\right), \ldots, \phi_{i 1}\left(a_{n}\right)\right)$ to the set of left generators $\operatorname{Lg}_{n}\left(A_{i}\right)$ is one for every $i$.

\section{Approximation and preimage}

Suppose that $M$ is a compact, connected and oriented differentiable manifold. Moreover, suppose that $L$ is a finite-dimensional, normed, real vector space and let $B$ denote the closed unit ball in $L$. Let $\pi_{1}$ and $\pi_{2}$ denote the coordinate projections from $B \times M$ to $B$ and $M$ respectively. Give $C(B \times M, L)$ the supremum norm and let $\mathcal{B}_{M}$ denote the open unit ball in $C(B \times M, L)$ with center $\pi_{1}$.

The following theorem is the main result in this section. Applying it with $L=\mathbb{C}^{n}$ (given the supremum norm) will eventually allow us to prove that $\operatorname{sr}(A)=n+1$. The more general version of Theorem 1, given here, will be useful when considering the real rank of $A$.

Theorem 1. There is a dense subset of $\mathcal{B}_{M}$ of functions $f$ such that if $\pi$ is the restriction of $\pi_{2}$ to $N:=f^{-1}\{0\}$, then $\pi^{*}: \mathrm{H}^{*}(M) \rightarrow \mathrm{H}^{*}(N)$ is injective.

Proposition 2. Let $\pi: N \rightarrow M$ be a map from a topological space $N$ to a compact orientable manifold of dimension $n$ and suppose that there exists an element $\nu \in$ $\mathrm{H}_{n}(N)$ such that $\pi_{*}(\nu)$ generates $\mathrm{H}_{n}(M)$. Then $\pi_{*}$ is surjective and $\pi^{*}$ is injective.

Proof. Note that it is implicit in the hypothesis that $M$ is connected. Put $\mu=\pi_{*}(\nu)$ and let $D_{\nu}: \mathrm{H}^{*}(N) \rightarrow \mathrm{H}_{*}(N)$ and $D_{\mu}: \mathrm{H}^{*}(M) \rightarrow \mathrm{H}_{*}(M)$ be given by cap product with $\nu$ and $\mu$ respectively. We will show that $D_{\mu}=\pi_{*} \circ D_{\nu} \circ \pi^{*}$. Let $\tilde{\nu}$ be an $n$-cycle in $N$ which represents $\nu$ and put $\tilde{\mu}=\pi_{*}(\tilde{\nu})$. For $a \in \mathrm{C}^{n-i}(M)$ and $b \in \mathrm{C}^{i}(N)$ we have

$$
\begin{aligned}
\left\langle a, \pi_{*}\left(\pi^{*}(b) \cap \tilde{\nu}\right)\right\rangle & =\left\langle\pi^{*}(a), \pi^{*}(b) \cap \tilde{\nu}\right\rangle=\left\langle\pi^{*}(a b), \tilde{\nu}\right\rangle \\
& =\left\langle a b, \pi_{*}(\tilde{\nu})\right\rangle=\langle a b, \tilde{\mu}\rangle=\langle a, b \cap \tilde{\mu}\rangle .
\end{aligned}
$$

Hence, $D_{\mu}=\pi_{*} \circ D_{\nu} \circ \pi^{*}$. By the Poincaré duality theorem $D_{\mu}$ is an isomorphism and the result follows.

Suppose that $\phi: N \rightarrow M$ is a map between two compact, oriented smooth manifolds of the same dimension and that $M$ is connected. One can then assign a number to $\phi$, called the degree, which is denoted by $\operatorname{deg}(\phi)-$ see [11]. If $\phi$ is differentiable, then we can describe the degree in the following way: Let $y \in M$ be a regular value for $\phi$. Then the preimage $\phi^{-1}\{y\}$ is finite. Now, for each point $x$ in the preimage the differential $d_{x} \phi$ is bijective and we give $x$ the sign +1 or -1 depending on whether the differential preserves or reverses the orientation. Then the sum of the signs of the points in the preimage is equal to the degree of $\phi$.

In the case of a map $\phi$ of a sphere to itself the degree is called the winding number and denoted by $\mathrm{W}(\phi)$. Another variation is when $\phi: \mathrm{S}^{n} \rightarrow \mathbb{R}^{n+1}$ and $y \in \mathbb{R}^{n+1}$ is not in the image of $\phi$. Then we let $\mathrm{W}(\phi, y)$ be the winding number of $\theta \circ \phi$ where $\theta: \mathbb{R}^{n+1}-\{y\} \rightarrow \mathrm{S}^{n}$ is given by $\theta(z)=(z-y) /|z-y|$.

If $M$ is an oriented manifold of dimension $n$, then the orientation gives a preferred generator $\mu_{x}$ for $\mathrm{H}_{n}(M, M-\{x\})$. Moreover, if $M$ is compact, there exists a unique element $\mu \in \mathrm{H}_{n}(M)$ - the fundamental homology class - such that for each $x \in M$ the natural map $\mathrm{H}_{n}(M) \rightarrow \mathrm{H}_{n}(M, M-\{x\})$ maps $\mu$ to $\mu_{x}$. 
Suppose that $\phi: N \rightarrow M$ is a map between compact oriented manifolds of the same dimension. Then $\pi_{*}(\nu)=\operatorname{deg}(\pi) \mu$, where $\nu \in \mathrm{H}_{n}(N)$ and $\mu \in \mathrm{H}_{n}(M)$ denote the fundamental homology classes. We therefore have the following:

Corollary 3. Suppose that $N, M$ are compact, oriented and differentiable manifolds of the same dimension and that $M$ is connected. If $\pi: N \rightarrow M$ is of degree $\operatorname{deg}(\pi)= \pm 1$, then $\pi^{*}$ is injective.

Proof of Theorem 1. Let $h \in \mathcal{B}_{M}$ and $\varepsilon>0$ be given. We may assume that $B(h, \varepsilon) \subseteq \mathcal{B}_{M}$. There exists a map $g: B \times M \rightarrow L$ which is smooth on the interior and such that $\|g-h\| \leq \varepsilon / 2$. By Sard's theorem, there exists a regular value $\omega$ for $g$ such that $|\omega| \leq \varepsilon / 2$; see for instance [11]. Then, zero is a regular value for $f:=g-\omega$ and $\|f-h\| \leq \varepsilon$. By the preimage theorem, $N:=f^{-1}\{0\}$ is a sub-manifold of $B \times M$ and it has the same dimension as $M$.

Choose some orientation of $L$ and let $\ell$ denote the dimension of $L$. We give $N$ the preimage orientation: For $y \in N$ the tangent space $\mathrm{T}_{y} N$ is the kernel of the differential $d_{y} f$. In particular, $\mathrm{T}_{y} N$ is a subspace of $\mathrm{T}_{y}(B \times M)$ of codimension $\ell$. Also, remember that $d_{y} f$ is surjective for $y \in N$. This means that if $V$ is an $\ell$-dimensional subspace of $\mathrm{T}_{y}(B \times M)$ which intersects $\mathrm{T}_{y} N$ at zero only, then $d_{y} f$ is an isomorphism on $V$, and so we get an orientation on $V$ from that on $\mathrm{T}_{0} L$ via $d_{y} f$. Now, orient $\mathrm{T}_{y} N$ so that the direct sum orientation of $\mathrm{T}_{y} N \oplus V$ coincides with that of $\mathrm{T}_{y}(B \times M)$. This gives an orientation of $N$. For more details, see page 100 of $[11]$.

Let $x \in M$ be a regular value for $\pi: N \rightarrow M$. Since $N$ and $M$ have the same dimension, the preimage $\pi^{-1}\{x\}$ is a zero-dimensional sub-manifold of $N$ and therefore a finite subset. Write $\pi^{-1}\{x\}=\left\{y_{1}, \ldots, y_{m}\right\}$. Let $w_{i}=+1$ or -1 depending on whether $d_{y_{i}} \pi$ preserves or reverses orientation. Then, by definition, $\sum_{i} w_{i}=\operatorname{deg}(\pi)$.

Now, $\left.d_{y_{i}} \pi_{2}\right|_{\mathrm{T}_{y_{i}}} N$ is equal to $d_{y_{i}} \pi$ which is surjective (because $x$ is a regular value for $\pi$ ) and therefore also injective. On the other hand, we have that $\pi_{2}$ maps the sub-manifold $B \times\{x\}$ to the point $x$ and therefore $\left.d_{y_{i}} \pi_{2}\right|_{\mathrm{T}_{y_{i}}(B \times\{x\})}=0$. It follows that the differential of $f$ at $y_{i}$ restricted to $\mathrm{T}_{y_{i}}(B \times\{x\})$ is an isomorphism onto $\mathrm{T}_{0} L$. Since $N$ is given the preimage orientation, the two isomorphisms $d_{y_{i}} \pi$ and $\left.d_{y_{i}} f\right|_{\mathrm{T}_{y_{i}}(B \times\{x\})}$ either both preserve or both reverse orientation.

Suppose that $\gamma_{i}: \mathrm{S}^{\ell-1} \rightarrow B \times\{x\} \backslash N$ is a map with winding number $\mathrm{W}\left(\gamma_{i}, y_{i}\right)=1$ around $y_{i}$ and $\mathrm{W}\left(\gamma_{i}, y_{j}\right)=0$ for $j \neq i$. By the inverse function theorem $\left.f\right|_{B \times\{x\}}$ is a diffeomorphism locally at $y_{i}$ and so $\mathrm{W}\left(f \circ \gamma_{i}, 0\right)= \pm 1$. In fact $\mathrm{W}\left(f \circ \gamma_{i}, 0\right)=$ +1 or -1 depending on whether $\left.d_{y_{i}} f\right|_{\mathrm{T}(B \times\{x\})}$ preserves or reverses orientation. Consequently, $w_{i}=\mathrm{W}\left(f \circ \gamma_{i}, 0\right)$.

Now, suppose that $\gamma: \mathrm{S}^{\ell-1} \rightarrow \partial B \times\{x\}$ is a homeomorphism with $\mathrm{W}(\gamma, 0)=1$. Then $\mathrm{W}(f \circ \gamma, 0)=\sum_{i} \mathrm{~W}\left(f \circ \gamma_{i}, 0\right)=\operatorname{deg}(\pi)$. On the other hand, $\left\|f-\pi_{1}\right\|<1$ and so we see that $\mathrm{W}(f \circ \gamma, 0)=1$. This shows that $\operatorname{deg}(\pi)=1$, and the result follows by Corollary 3 .

The following corollary will make it possible to show that the real rank of $A$ is at least $n$. Let $\pi_{1}$ and $\pi_{2}$ denote the coordinate projections from $\mathbb{D}^{n} \times M$ to $\mathbb{D}^{n}$ and $M$ respectively. Let Re $: \mathbb{C}^{n} \rightarrow \mathbb{R}^{n}$ take the real part of each coordinate and let $\mathcal{B}_{M}$ denote the open unit ball in $C\left(\mathbb{D}^{n} \times M, \mathbb{R}^{n}\right)$ with center $\operatorname{Re} \circ \pi_{1}$.

Corollary 4. There is a dense subset of $\mathcal{B}_{M}$ of functions $f$ such that if $\pi$ is the restriction of $\pi_{2}$ to $N:=f^{-1}\{0\}$, then $\pi^{*}: \mathrm{H}^{*}(M) \rightarrow \mathrm{H}^{*}(N)$ is injective. 
Proof. Let $h \in \mathcal{B}_{M}$ and $\varepsilon>0$ be given. We may assume that $B(h, \varepsilon) \subseteq \mathcal{B}_{M}$. Applying Theorem 1 in the case where $L=\mathbb{R}^{n}$ with the supremum norm it follows that there exists a map $g:[-1,1]^{n} \times M \rightarrow \mathbb{R}^{n}$ which is within $\varepsilon$ of $h$ on $[-1,1]^{n} \times M$ and such that if $\tilde{\pi}$ is the restriction of $\pi_{2}$ to $\tilde{N}:=g^{-1}\{0\}$, then $\tilde{\pi}^{*}: \mathrm{H}^{*}(M) \rightarrow \mathrm{H}^{*}(\tilde{N})$ is injective. Let $\delta>0$ be such that if $(v, x) \in \mathbb{D}^{n} \times M$ and $|\operatorname{Im}(v)|<\delta$, then $|h(v, x)-h(\operatorname{Re}(v), x)|<\varepsilon$. Now, put

$$
f(v, x)=\alpha(v, x) h(v, x)+(1-\alpha(v, x)) g(\operatorname{Re}(v), x),
$$

where $\alpha(v, x)=\max \left\{\delta^{-1}|\operatorname{Im}(v)|, 1\right\}$. Then $f$ is continuous, $\|f-h\| \leq \varepsilon$ and $f \in \mathcal{B}_{M}$. Moreover, if we put $N=f^{-1}\{0\}$, then $N \cap[-1,1]^{n} \times M=\tilde{N}$. If we let $\iota$ denote the inclusion $\tilde{N} \subseteq N$, then $\tilde{\pi}=\pi \circ \iota$ and therefore $\tilde{\pi}^{*}=\iota^{*} \circ \pi^{*}$. It follows that $\pi^{*}$ is injective.

\section{Certain elements Which are far from the left generators}

Suppose that $B$ is a unital $\mathrm{C}^{*}$-algebra and that $p \in B$ is a projection. Then we write $p^{\perp}$ for the orthogonal complement $1-p$ of $p$ in $B$. For $k$ a natural number we write $k p$ for the $k$-fold direct sum of $p$ with itself in $B \otimes \mathcal{K}$. This is determined up to equivalence. If $q$ is a projection in $B$ which is equivalent to a sub-projection of $p$, then we write $q \precsim p$. We will use the same notation for vector bundles.

Proposition 5. Suppose that $B$ is a unital $C^{*}$-algebra and $p \in B$ is a projection. If there exists $\left(b_{1}, \ldots, b_{k}\right) \in \operatorname{Lg}_{k}(B)$ such that $p b_{i} p=0$ for all $i$, then $p \precsim k p^{\perp}$.

Proof. By assumption there exist $c_{1}, \ldots, c_{k} \in B$ such that $\sum_{i} c_{i} b_{i}=1$. Let $\left(e_{i j}\right)$ be a system of matrix units for $\mathrm{M}_{k}$, and consider, in $B \otimes \mathrm{M}_{k}$, the elements $b=$ $\sum_{i} b_{i} \otimes e_{i 1}, \tilde{p}=p \otimes e_{11}, c=\sum_{j} c_{j} \otimes e_{1 j}$ and $\tilde{q}=p^{\perp} \otimes 1$. Note that $\tilde{q} b \tilde{p}=b \tilde{p}$ and that $c b=1 \otimes e_{11}$. Put $x=\tilde{p} c \tilde{q}$ and $y=\tilde{q} b \tilde{p}$. Then $x y=\tilde{p} c \tilde{q} b \tilde{p}=\tilde{p} c b \tilde{p}=\tilde{p}$ and $y x=\tilde{q} b \tilde{p} c \tilde{q} \in\left(B \otimes \mathrm{M}_{k}\right) \tilde{q}$. So $\tilde{p} \precsim \tilde{q}$.

Let $B$ denote a homogeneous $\mathrm{C}^{*}$-algebra. By [10, Theorem 3.2], there exists a locally trivial $\mathrm{M}_{m}(\mathbb{C})$-bundle $\mu$ such that $B \cong \Gamma(\mu)$ - the $\mathrm{C}^{*}$-algebra of sections in $\mu$. The base-space of $\mu$ can be taken to be $\hat{B}-$ the spectrum of $B$. For $N \subseteq \hat{B}$ a closed subset let $I_{N}$ denote the ideal in $B$ consisting of sections in $\mu$ which vanish on $N$. Led by the fact that $B / I_{N} \cong \Gamma\left(\left.\mu\right|_{N}\right)$ we let $\left.b\right|_{N}$ denote the image of $b$ in $B / I_{N}(b \in B)$.

Corollary 6. Suppose that $B$ is a unital homogeneous $C^{*}$-algebra, $p \in B$ is a projection and $\left(b_{1}, \ldots, b_{k}\right) \in \operatorname{Lg}_{k}(B)$. Then $\left.\left.p\right|_{N} \precsim k p\right|_{N} ^{\perp}$ where $N$ is the zero set of $\left(p b_{1} p, \ldots, p b_{k} p\right)$.

Suppose that $\eta$ is a complex vector bundle over $M$ with $\mathrm{e}(\eta)^{n} \neq 0(M$ as in section 4) and suppose that $\left.B=\left(C\left(\mathbb{D}^{n} \times M\right) \otimes \mathcal{K}\right)\right)_{p+q}$ where $p$ and $q$ are orthogonal projections which correspond to $\theta_{1}$ and $\pi_{2}^{*}(\eta)$ respectively. Let $a_{j} \in C\left(\mathbb{D}^{n}\right)$ be projection onto the $j$ 'th coordinate, and let $\pi_{1}$ be projection of $\mathbb{D}^{n} \times M$ onto $\mathbb{D}^{n}$.

Theorem 7. Suppose that $b_{1}, \ldots, b_{n} \in B$ are such that $p b_{j} p=\left(a_{j} \circ \pi_{1}\right) p$ for each $j$. Then the distance from $\left(b_{1}, \ldots, b_{n}\right)$ to $\operatorname{Lg}_{n}(B)$ is at least one.

Proof. Put $b=\left(b_{1}, \ldots, b_{n}\right)$ and $\tilde{p}=(p, \ldots, p) \in B^{n}$. Assume that there exists an element $c \in \operatorname{Lg}_{n}(B)$ such that $\|b-c\|<1$. Since $\tilde{p}$ is a projection of rank one, there exists a function $g \in C\left(\mathbb{D}^{n} \times M\right)^{n}$ such that $\tilde{p} c \tilde{p}=g \tilde{p}$. Let $\mathcal{B}_{M}$ be as in the previous section where $L=\mathbb{C}^{n}$ with the supremum norm. Then $g \in \mathcal{B}_{M}$ because $\tilde{p} b \tilde{p}=\pi_{1} \cdot \tilde{p}$. 
Now, let $\varepsilon>0$ such that $B(c, \varepsilon) \subseteq \operatorname{Lg}_{n}(B)$ and $B(g, \varepsilon) \subseteq \mathcal{B}_{M}$. Let $f \in B(g, \varepsilon)$ be as in Theorem 1. Consider the element $d=c+(f-g) p$. Note that $\tilde{p} d \tilde{p}=f \tilde{p}$. Moreover, $d \in \operatorname{Lg}_{n}(B)$ because $\|d-c\|<\varepsilon$. By Corollary 6 , we have that $\left.\left.p\right|_{N} \precsim n q\right|_{N}$, where $N=f^{-1}\{0\}$. In terms of bundles this means that $\left.\theta_{1} \precsim n \pi_{2}^{*}(\eta)\right|_{N}$. But this, we will show, cannot be. If $\iota$ denotes the inclusion of $N$ in $\mathbb{D}^{n} \times M$, then $\pi=\pi_{2} \circ \iota$ is the restriction of $\pi_{2}$ to $N$ and $\pi^{*}(\eta)=\iota^{*}\left(\pi_{2}^{*}(\eta)\right)=\left.\pi_{2}^{*}(\eta)\right|_{N}$. It follows that $\theta_{1} \precsim n \pi^{*}(\eta)$ and so the Euler class $e\left(n \pi^{*}(\eta)\right)$ is zero. But $e\left(n \pi^{*}(\eta)\right)=\pi^{*}\left(e(\eta)^{n}\right)$, $e(\eta)^{n}$ is nonzero and $\pi^{*}: \mathrm{H}^{*}(M) \rightarrow \mathrm{H}^{*}(N)$ is injective by Theorem 1 . This is a contradiction and we conclude that $\operatorname{dist}\left(\left(b_{1}, \ldots, b_{n}\right), \operatorname{Lg}_{n}(B)\right) \geq 1$.

\section{The stable Rank of $A$}

Theorem 8. The stable rank of $A$ is $n+1$.

Proof. Note that $\phi_{i}=\psi_{i} \oplus \chi_{i}$, where $\psi_{i}$ comes from $\left(\pi_{i+1}^{1}, \theta_{1}\right)$ and $\chi_{i}$ comes from $\left(y_{i}^{j}, \zeta_{i+1}\right)_{j}$. Now $\psi_{i 0}\left(p_{0}\right)$ is a sub-projection of $p_{i}$ which corresponds to a trivial line bundle. Therefore $p_{i}-\psi_{i 0}\left(p_{0}\right)$ corresponds to $\xi_{i} \ominus \theta_{1}$, and we saw earlier that $\mathrm{e}\left(\xi_{i} \ominus \theta_{1}\right)^{n} \neq 0$. Furthermore,

$$
\psi_{i 0}\left(p_{0}\right) \phi_{i 0}\left(a_{j}\right) \psi_{i 0}\left(p_{0}\right)=\psi_{i 0}\left(a_{j}\right)=\left(a_{j} \circ \pi_{i}\right) \psi_{i 0}\left(p_{0}\right),
$$

where $\pi_{i}: X_{i} \rightarrow \mathbb{D}^{n}$ is projection onto $\mathbb{D}^{n}$. By Theorem 7 , the distance from $\left(\phi_{i 0}\left(a_{1}\right), \ldots, \phi_{i 0}\left(a_{n}\right)\right)$ to $\operatorname{Lg}_{n}\left(A_{i}\right)$ is one for every $i$. It follows that the distance from $\left(\phi_{\infty 0}\left(a_{1}\right), \ldots, \phi_{\infty 0}\left(a_{n}\right)\right)$ to $\operatorname{Lg}_{n}(A)$ is one. So $\operatorname{Lg}_{n}(A)$ is not dense in $A^{n}$ and therefore $\operatorname{sr}(A) \geq n+1$.

From [15] we have the following formula for the stable rank of a unital $\mathrm{m}$ homogeneous algebra $B$ :

$$
\operatorname{sr}(B)=\lceil\lfloor\operatorname{dim}(\hat{B}) / 2\rfloor / m\rceil+1,
$$

where $\hat{B}$ denotes the spectrum of $B,\lceil t\rceil$ denotes the least integer greater than $t$ and $\lfloor t\rfloor$ denotes the largest integer less than $t$.

With $\sigma(0)=1$ we have $\operatorname{dim}\left(X_{i}\right)=2 n \sum_{j=0}^{i} \sigma(j)$ and one can easily show that $\sum_{j=0}^{i} \sigma(j)=(i+1)$ !. So $\operatorname{dim}\left(X_{i}\right)=2 n \operatorname{rank}\left(p_{i}\right)$ and it follows that $\operatorname{sr}\left(A_{i}\right)=n+1$. From Theorem 5.1 of $[16]$ we have $\operatorname{sr}(A) \leq n+1$.

It is a well-known fact that a $\mathrm{C}^{*}$-algebra $B$ with stable rank one has cancellation of projections, i.e. if $p, q, r$ are projections in $B \otimes \mathcal{K}$ and $p \oplus r \sim q \oplus r$, then $p \sim q$. It is therefore natural to ask whether $A$ has this property.

Note that if $\sigma$ is a trace on $A_{i+1}$, then $\sigma \circ \phi_{i}$ is the average of one trace which depends on $\sigma$ and $i+1$ traces which do not depend on $\sigma$ (corresponding to the point evaluations). Using this, one can show that $A$ has a unique tracial state.

\section{The Real Rank of $A$}

In [6] the notion of real rank was introduced. The real rank of a unital $C^{*}$-algebra $B$, denoted by $\operatorname{RR}(B)$, is the least integer $k$ such that $\operatorname{Lg}_{k+1}(B) \cap B_{\mathrm{sa}}^{k+1}$ is dense in $B_{\mathrm{sa}}^{k+1}$ where $B_{\mathrm{sa}}$ denotes the set of self-adjoint elements in $B$.

We will show here that the above method gives the lower bound $\operatorname{RR}(A) \geq n$. The formula of Beggs and Evans [1] for the real rank of a homogeneous $\mathrm{C}^{*}$-algebra gives the upper bound $\operatorname{RR}(A) \leq n+1$. So $\operatorname{RR}(A)$ is either equal to or one less than $\operatorname{sr}(A)$. 
With the same notation as in Theorem 7 we have the following:

Theorem 9. Suppose that $b_{1}, \ldots, b_{n} \in B_{\mathrm{sa}}$ and $p b_{j} p=\left(\operatorname{Re}\left(a_{j}\right) \circ \pi_{1}\right) p$ for every $j$. Then the distance from $\left(b_{1}, \ldots, b_{n}\right)$ to $\operatorname{Lg}_{n}(B) \cap B_{\mathrm{sa}}^{n}$ is at least one.

Proof. Assume that there exists $c \in \operatorname{Lg}_{n}(B) \cap B_{\mathrm{sa}}^{n}$ such that $\|b-c\|<1$, where $b=\left(b_{1}, \ldots, b_{n}\right)$. Put $\tilde{p}=(p, \ldots, p) \in B^{n}$. Since $c$ is self-adjoint, $\tilde{p} c \tilde{p}$ is of the form $g \tilde{p}$ for some $g \in C\left(\mathbb{D}^{n} \times M, \mathbb{R}^{n}\right)$. As in the proof of Theorem 7 it follows that there exists $d \in \operatorname{Lg}_{n}(B) \cap B_{\mathrm{sa}}^{n}$ such that $\tilde{p} d \tilde{p}=f \tilde{p}$ where $f$ is as in Corollary 4. By Corollary 6 we have $\left.\left.p\right|_{N} \precsim n q\right|_{N}$, where $N=f^{-1}\{0\}$. Exactly as in the proof of Theorem 7 we reach a contradiction with the assumption that the Euler class of $n \eta$ is nonzero.

Theorem 10. The real rank of $A$ is either $n$ or $n+1$.

Proof. The proof that $\operatorname{RR}(A) \geq n$ is the same as in Theorem 8 but this time with the tuple $\left(\operatorname{Re}\left(a_{1}\right), \ldots, \operatorname{Re}\left(a_{n}\right)\right)$ and using Theorem 9 in place of Theorem 7 .

If $B$ is a unital trivial $m$-homogeneous $C^{*}$-algebra, then from [1] we have the formula

$$
\operatorname{RR}(B)=\left\lceil\frac{\operatorname{dim}(\hat{B})}{2 m-1}\right\rceil
$$

Arguing as in [15], one can show that this holds for a general unital $m$-homogeneous $\mathrm{C}^{*}$-algebra. So $\mathrm{RR}\left(A_{i}\right)=\left\lceil 2 n \operatorname{rank}\left(p_{i}\right) /\left(2 \operatorname{rank}\left(p_{i}\right)-1\right)\right\rceil$. The latter is seen to be $n+1$ for large $i$. It follows that $\operatorname{RR}(A) \leq n+1$.

\section{The ordered $K_{0}$-Group of $A$}

We will show that $A$ has perforation, i.e. that $\mathrm{K}_{0}(A)$ is not weakly unperforated. Remember that a simple, partially ordered abelian group $G$ is said to be weakly unperforated if $\ell x>0$ implies $x>0$ for $x \in G, \ell \in \mathbb{N}$. In [20] examples were given of simple $\mathrm{C}^{*}$-algebras with perforation.

Notation. For $(G, u)$ a simple, partially ordered abelian group with order unit, let $G^{(0, u)}$ denote the set of $x \in G$ for which $0<x<u$, and let

$$
\kappa(x)=\min \left\{\ell \in \mathbb{N}: x \leq \ell x^{\perp}\right\}
$$

for $x \in G^{(0, u)}$, where $x^{\perp}=u-x$.

Note that if $x \leq y$, then $\kappa(x) \leq \kappa(y)$ and that if $\alpha:(G, u) \rightarrow(H, v)$ is a homomorphism with $\alpha(u)=v$, then $\kappa(\alpha(x)) \leq \kappa(x)$.

The choice of order unit in $\mathrm{K}_{0}(A)$ is, as usual, the class of the unit $1=\left[1_{A}\right]$. In the following, $\sigma$ denotes the unique state on $\mathrm{K}_{0}(A)$.

Proposition 11. (i) If $x \in \mathrm{K}_{0}(A)^{(0,1)}$ and $\sigma(x) \leq(n+2)^{-1}$, then $\kappa(x) \leq n+1$.

(ii) There exists a decreasing sequence $\left(x_{i}\right)_{i=1}^{\infty}$ in $\mathrm{K}_{0}(A)^{(0,1)}$ with $\lim _{i \rightarrow \infty} \sigma\left(x_{i}\right)=$ 0 such that $\kappa\left(x_{i}\right)=n+1$ for all $i$.

In contrast to this, if $G$ is weakly unperforated, $x \in G^{(0,1)}$ and $\sigma(x)<\frac{1}{2}$ for every state $\sigma$, then $\kappa(x)=1$. 
Proof. (i) Let $i \in \mathbb{N}$ and $p \in A_{i}$ be a projection with $x=\left[\phi_{\infty i}(p)\right]$. Since every $\phi_{j}$ is unital we have $\sigma(x)=\frac{\operatorname{rank}(p)}{\operatorname{rank}\left(p_{i}\right)}$ and therefore $\operatorname{rank}\left(p_{i}\right) \geq(n+2) \operatorname{rank}(p)$. Hence,

$$
\begin{aligned}
(n+1) \operatorname{rank}\left(p^{\perp}\right)-\operatorname{rank}(p) & =(n+1) \operatorname{rank}\left(p_{i}\right)-(n+2) \operatorname{rank}(p) \\
& \geq n \operatorname{rank}\left(p_{i}\right) \\
& =\frac{\operatorname{dim}\left(X_{i}\right)}{2} .
\end{aligned}
$$

It follows from [13, Theorem 9.1.2] that $[p] \leq(n+1)\left[p^{\perp}\right]$ and therefore $\kappa([p]) \leq n+1$.

(ii) With notation as in the proof of Theorem 8 , put $x_{i}=\left[\phi_{\infty i} \circ \psi_{i 0}\left(p_{0}\right)\right]$. Clearly, this is a decreasing sequence. Now, $\psi_{i 0}\left(p_{0}\right)$ corresponds to a trivial line bundle and its orthogonal complement, $p_{i}-\psi_{i 0}\left(p_{0}\right)$, corresponds to $\xi_{i} \ominus \theta_{1}$. The element in $\mathrm{K}^{0}\left(X_{i}\right)$ corresponding to $n\left[\psi_{i 0}\left(p_{0}\right)^{\perp}\right]-\left[\psi_{i 0}\left(p_{0}\right)\right]$ is therefore $n\left[\xi_{i} \ominus \theta_{1}\right]-\left[\theta_{1}\right]$.

Suppose, for a moment, that $n\left[\xi_{i} \ominus \theta_{1}\right]-\left[\theta_{1}\right]$ is positive. Then there exist $d \in \mathbb{N}$ and a vector bundle $\eta$ such that $n\left(\xi_{i} \ominus \theta_{1}\right) \oplus \theta_{d} \cong \eta \oplus \theta_{d+1}$. For $\omega$ a complex vector bundle let $c_{k}(\omega)$ denote the $k^{\prime}$ th Chern class. If $m$ denotes the fiber dimension of $\xi_{i} \ominus \theta_{1}$, then

$$
\mathrm{e}\left(n\left(\xi_{i} \ominus \theta_{1}\right)\right)=\mathrm{c}_{n m}\left(n\left(\xi_{i} \ominus \theta_{1}\right)\right)=\mathrm{c}_{n m}(\eta),
$$

because the Chern classes are invariant under stable equivalence [14, Lemma 14.3]. Now, $\mathrm{c}_{n m}(\eta)=0$ because $\eta$ has fiber dimension (one) less than $n m$. But

$$
\mathrm{e}\left(n\left(\xi_{i} \ominus \theta_{1}\right)\right)=\mathrm{e}\left(\xi \ominus \theta_{1}\right)^{n}
$$

is nonzero. This is a contradiction. So $n\left[\xi_{i} \ominus \theta_{1}\right]-\left[\theta_{1}\right]$ is nonpositive and therefore $\kappa\left(\left[\psi_{i 0}\left(p_{0}\right)\right]\right)>n$. Since $\left[\phi_{j i} \circ \psi_{i 0}\left(p_{0}\right)\right] \geq\left[\psi_{j 0}\left(p_{0}\right)\right]$ we have that $\kappa\left(\left[\phi_{j i} \circ \psi_{i 0}\left(p_{0}\right)\right]\right)>n$ for every $j>i \geq 1$. It follows that $\kappa\left(x_{i}\right)>n$.

\section{INFINITE STABLE RANK}

Suppose that the construction considered so far is changed in the following way. The space $X_{i}$ is replaced by

$$
X_{i}=\mathbb{D}^{i \sigma(i)^{2}} \times \mathbb{C P}^{\sigma(1)} \times \mathbb{C P}^{2 \sigma(2)} \times \cdots \times \mathbb{C} \mathrm{P}^{i \sigma(i)} .
$$

Then $X_{i+1}=\mathbb{D}^{(i+1) \sigma(i+1)^{2}-i \sigma(i)^{2}} \times X_{i} \times \mathbb{C P}^{(i+1) \sigma(i+1)}$ and we let $\pi_{i+1}^{1}$ and $\pi_{i+1}^{2}$ denote the coordinate projections from $X_{i+1}$ onto $X_{i}$ and $\mathbb{C P}^{(i+1) \sigma(i+1)}$ respectively. The $*$-homomorphism $\phi_{i}$ is changed accordingly by letting $\zeta_{i}=\pi_{i}^{2 *}\left(\gamma_{i \sigma(i)}\right)$.

Theorem 12. The stable rank of $A$ is infinite.

Proof. As before, $\xi_{i}$ denotes the bundle corresponding to $p_{i}$ - the unit of $A_{i}$. Let $\pi_{i j}=\pi_{i+1} \circ \ldots \circ \pi_{j}: X_{j} \rightarrow X_{i}$. To ease the notation, denote $\pi_{j i}^{*}\left(\zeta_{i}\right)$ by $\zeta_{i}$ also. From the definition of $\phi_{i}$ we see that

$$
\xi_{i} \cong \theta_{1} \oplus \sigma(1) \zeta_{1} \oplus \sigma(2) \zeta_{2} \oplus \cdots \oplus \sigma(i) \zeta_{i}
$$

and so there exist mutually orthogonal projections $q_{1}, \ldots, q_{\sigma(i)} \in A_{i}$ each of which corresponds to the bundle $\zeta_{i}$. Let $\rho_{k \ell s}: X_{i} \rightarrow \mathbb{D}, 1 \leq k, \ell \leq \sigma(i), 1 \leq s \leq i$, be an indexing of the projections onto the coordinates of the factor $\mathbb{D}^{i \sigma(i)^{2}}$ in $X_{i}$. Let $e_{1 \ell} \in A_{i}$ such that $e_{1 \ell} e_{1 \ell}^{*}=q_{1}$ and $e_{1 \ell}^{*} e_{1 \ell}=q_{\ell}$ and put $e_{k \ell}=e_{1 k}^{*} e_{1 \ell}$ for $1 \leq k, \ell \leq \sigma(i)$ and let

$$
a_{s}=\sum_{1 \leq k, \ell \leq \sigma(i)} \rho_{k \ell s} e_{k \ell}, \quad 1 \leq s \leq i .
$$


Now fix $j \geq i$ and let $a=\left(\phi_{j i}\left(a_{1}\right), \ldots, \phi_{j i}\left(a_{i}\right)\right)$. We will show that the distance from $a$ to $\operatorname{Lg}_{i}\left(A_{j}\right)$ is at least one. Write $\phi_{i}=\psi_{i} \oplus \chi_{i}$ in the same way as in the proof of Theorem 8. Then $\psi_{j i}\left(e_{\ell \ell}\right)$ corresponds to $\zeta_{i}$ for all $1 \leq \ell \leq \sigma(i)$ and

$$
\begin{aligned}
\psi_{j i}\left(e_{k k}\right) \phi_{j i}\left(a_{s}\right) \psi_{j i}\left(e_{\ell \ell}\right) & =\psi_{j i}\left(\rho_{k \ell s} e_{k \ell}\right) \\
& =\left(\rho_{k \ell s} \circ \pi_{i j}\right) \psi_{j i}\left(e_{k \ell}\right) .
\end{aligned}
$$

Letting $\tilde{e}_{k \ell}=\left(\psi_{j i}\left(e_{k \ell}\right), \ldots, \psi_{j i}\left(e_{k \ell}\right)\right) \in A_{j}^{i}$ we can rewrite this as

$$
\tilde{e}_{k k} a \tilde{e}_{\ell \ell}=\left(\rho_{k \ell 1} \circ \pi_{i j}, \ldots, \rho_{k \ell i} \circ \pi_{i j}\right) \tilde{e}_{k \ell} .
$$

Let $\rho: X_{j} \rightarrow \mathbb{D}^{i \sigma(i)^{2}}$ be the coordinate projection which has all $\rho_{k \ell s} \circ \pi_{i j}$ as coordinate functions. Now, suppose there exists $b \in \operatorname{Lg}_{i}\left(A_{j}\right)$ such that $\|a-b\|<1$. Since $\tilde{e}_{\ell \ell}$ is a projection of rank one, there exist maps $g_{k \ell s}: X_{j} \rightarrow \mathbb{C}$ such that $\tilde{e}_{k k} b \tilde{e}_{\ell \ell}=\left(g_{k \ell 1}, \ldots, g_{k \ell i}\right) \tilde{e}_{k \ell}$. Let $g: X_{j} \rightarrow \mathbb{D}^{i \sigma(i)^{2}}$ be the map with all these $g_{k \ell s}$ as coordinate functions ordered in the same way as $\rho_{k \ell s} \circ \pi_{i j}$ in $\rho$. Then $\|g-\rho\|<1$. In the same way as in the proof of Theorem 7 we can assume that restriction to $N=g^{-1}\{0\}$ induces an injection on cohomology. From Corollary 6 it follows that $\left.\left.\sigma(i) \zeta_{i}\right|_{N} \precsim i\left(\xi_{j} \ominus \sigma(i) \zeta_{i}\right)\right|_{N}$. In particular, $\left.\left.\zeta_{i}\right|_{N} \precsim i\left(\xi_{j} \ominus \sigma(i) \zeta_{i}\right)\right|_{N}$ and tensoring this by the dual of $\zeta_{i}$ we get $\left.\theta_{1} \precsim i\left(\left(\xi_{j} \ominus \sigma(i) \zeta_{i}\right) \otimes \zeta_{i}^{*}\right)\right|_{N}$. The above mentioned property of $N \subseteq X_{j}$ ensures that the Euler class of $i\left(\xi_{j} \ominus \sigma(i) \zeta_{i}\right) \otimes \zeta_{i}^{*}$ is zero.

With $\zeta_{0}=\theta_{1}$ and $\sigma(0)=1$ we have

$$
\begin{aligned}
\mathrm{e}\left(i\left(\xi_{j} \ominus \sigma(i) \zeta_{i}\right) \otimes \zeta_{i}^{*}\right) & =\mathrm{e}\left(i\left(\sigma(0) \zeta_{0} \oplus \sigma(1) \zeta_{1} \oplus \cdots \oplus \widehat{\sigma(i) \zeta_{i}} \oplus \cdots \oplus \sigma(j) \zeta_{j}\right) \otimes \zeta_{i}^{*}\right) \\
& =\prod_{\substack{0 \leq \ell \leq j \\
\ell \neq i}}\left(\mathrm{e}\left(\zeta_{\ell}\right)-\mathrm{e}\left(\zeta_{i}\right)\right)^{i \sigma(\ell)} \\
& =\sum_{\substack{0 \leq t_{0}, t_{1}, \ldots, \hat{t}_{i}, \ldots, t_{j} \\
t_{\ell} \leq i \sigma(\ell)}}\left(-\mathrm{e}\left(\zeta_{i}\right)\right)^{\sum_{\ell} t_{\ell}} \prod_{\substack{0 \leq \ell \leq j \\
\ell \neq i}} \mathrm{e}\left(\zeta_{\ell}\right)^{i \sigma(\ell)-t_{\ell}} .
\end{aligned}
$$

Since the bundles $\zeta_{1}, \ldots, \zeta_{j}$ vary over distinct factors of $X_{j}$, it follows from the Künneth formula that the terms of the above sum belong to distinct summands and therefore if one of the terms is nonzero then so is the sum. The term where $t_{\ell}$ is zero for $\ell>i$ and equal to $i \sigma(\ell)$ for $\ell<i$ is

$$
\mathrm{e}\left(\zeta_{i}\right)^{\sigma(i)} \prod_{\ell=i+1}^{j} \mathrm{e}\left(\zeta_{\ell}\right)^{i \sigma(\ell)}
$$

because $i \sum_{\ell=0}^{i-1} \sigma(\ell)=\sigma(i)$. It follows from the Künneth formula that this term is nonzero because each $\zeta_{\ell}$ was chosen such that $\mathrm{e}\left(\zeta_{\ell}\right)^{\ell \sigma(\ell)}$ is nonzero. So, in fact, $\mathrm{e}\left(i\left(\xi_{j} \ominus \sigma(i) \zeta_{i}\right) \otimes \zeta_{i}^{*}\right)$ is nonzero. We conclude therefore that $\operatorname{dist}\left(a, \operatorname{Lg}_{i}\left(A_{j}\right)\right) \geq 1$.

Theorem 13. The real rank of $A$ is infinite.

Proof. In the notation of the above proof put

$$
h_{s}=\sum_{k=1}^{\sigma(i)} \operatorname{Re}\left(\rho_{k k s}\right) e_{k k s}+\sum_{1 \leq k<\ell \leq \sigma(i)}\left(\rho_{k \ell s} e_{k \ell}+\bar{\rho}_{k \ell s} e_{\ell k}\right), \quad 1 \leq s \leq i .
$$

Then $\left(\phi_{j i}\left(h_{1}\right), \ldots, \phi_{j i}\left(h_{i}\right)\right)$ has distance at least one to $\operatorname{Lg}_{i}\left(A_{j}\right) \cap\left(A_{j}\right)_{\mathrm{sa}}^{i}$. The proof is a modification of that of Theorem 12 in the same way that the proof of Theorem 8 is modified to prove Theorem 10. 
Suppose $B$ is a $\mathrm{C}^{*}$-algebra containing a sequence $s_{1}, s_{2}, \ldots$ of isometries with mutually orthogonal ranges. From [16] we know that $B$ has infinite stable rank. Let $c=c_{1}, c_{2}, \ldots$ be a sequence of positive real numbers. A simple reorganisation of the argument found in [16] then yields:

Proposition 14. $\operatorname{dist}\left(\left(c_{1} s_{1}^{*}, \ldots, c_{n} s_{n}^{*}\right), \operatorname{Lg}_{n}(B)\right) \geq\left(\sum_{i=1}^{n} c_{i}^{-1}\right)^{-1}, n \in \mathbb{N}$.

Proof. Suppose that there exist $n \in \mathbb{N}$ and $\left(a_{1}, \ldots, a_{n}\right),\left(b_{1}, \ldots, b_{n}\right) \in B^{n}$ such that $\sum_{j=1}^{n} b_{j} a_{j}=1$ and $\left\|c_{j} s_{j}^{*}-a_{j}\right\|<\left(\sum_{i=1}^{n} c_{i}^{-1}\right)^{-1}$ for every $1 \leq j \leq n$. Put $p_{0}=1-\sum_{i=1}^{n} s_{i} s_{i}^{*}$ and $d=c_{1}^{-1} s_{1} a_{1}+\cdots+c_{n}^{-1} s_{n} a_{n}+p_{0}$. Then

$$
\|1-d\| \leq \sum_{i=1}^{n}\left\|s_{i} s_{i}^{*}-c_{i}^{-1} s_{i} a_{i}\right\|=\sum_{i=1}^{n} c_{i}^{-1}\left\|c_{i} s_{i}^{*}-a_{i}\right\|<1
$$

so $d$ is invertible. We can also write $d=\sum_{i=1}^{n}\left(c_{i}^{-1} s_{i}+p_{0} b_{i}\right) a_{i}$. If we multiply this on the left by $c_{i} s_{i}^{*}$ we find that $c_{i} s_{i}^{*} d=a_{i}$ and if we multiply on the right by $d^{-1} p_{0}$ we find that $p_{0}=0$, which is a contradiction.

In particular, every simple, infinite $\mathrm{C}^{*}$-algebra $B$ contains a sequence $a_{1}, a_{2}, \ldots$ for which $\operatorname{dist}\left(\left(a_{1}, \ldots, a_{n}\right), \operatorname{Lg}_{n}(B)\right), n=1,2,3, \ldots$, is bounded away from zero. It seems likely that no such sequence can be found in the algebra $A$ constructed above.

\section{REFERENCES}

[1] Beggs, E. J. and Evans, D. E., The real rank of algebras of matrix valued functions, Internat. J. Math., 2, (1991), pp. 131-138. MR 92e:46114

[2] Blackadar, B., A stable cancellation theorem for simple $\mathrm{C}^{*}$-algebras, Proc. London Math. Soc., (3), 47, (1983), pp. 303-305. MR 85g:46086

[3] Blackadar, B., Comparison theory for simple $\mathrm{C}^{*}$-algebras, Operator Algebras and Applications, L.M.S. Lecture Notes Series, 135, (1989), pp. 21-54. MR 90g:46078

[4] Blackadar, B., Dădărlat, M., and Rørdam, M., The real rank of inductive limit C*-algebras, Math. Scand, 69, (1991), pp. 211-216. MR 93e:46067

[5] Blackadar, B., Kumjian, A. and Rørdam, M., Approximately central matrix units and the structure of noncommutative tori, K-theory, 6, (1992), pp. 267-284. MR 93i:46129

[6] Brown, L. and Pedersen, G. K., C* algebras of real rank zero, J. Funct. Anal., 99, (1991), pp. 131-149. MR 92m:46086

[7] Cuntz, J., K-theory for certain C*-algebras, Ann. of Math., 113, (1981), pp. 181-197. MR 84c: 46058

[8] Dădărlat, M., Nagy, G., Némethi, A. and Pasnicu, C., Reduction of topological stable rank in inductive limits of $\mathrm{C}^{*}$-algebras, Pacific J. Math., 153, (1992), pp. 267-276. MR 93d:46119

[9] Dold, A., Lectures on Algebraic Topology, Springer-Verlag, 1972. MR 54:3685

[10] Fell, J. M. G., The structure of algebras of operator fields, Acta. math., 106, (1961), pp. 233280. MR 29:1547

[11] Guillemin, V. and Pollack, A., Differential topology, Prentice-Hall, New Jersey, 1974. MR 50:1276

[12] Herman, R. H. and Vaserstein, L. N., The stable range of $\mathrm{C}^{*}$-algebras, Invent. math., $\mathbf{7 7}$, (1984), pp. 553-555. MR 86a:46074

[13] Husemoller, D., Fibre Bundles, McGraw-Hill, New York, 1966. MR 37:4821

[14] Milnor, J. W. and Stasheff, J. D., Characteristic Classes, Annals of Mathematics Studies, No.76, Princeton University Press, 1974. MR 55:13428

[15] Nistor, V., Stable rank for a certain class of type I C*-algebras, J. Operator Theory, 17, (1987), pp. 365-373. MR 88h:46110

[16] Rieffel, M. A., Dimension and stable rank in the K-theory of $\mathrm{C}^{*}$-algebras, Proc. London Math. Soc. (3), 46, (1983), pp. 301-333. MR 84g:46085

[17] Rieffel, M. A., The cancellation theorem for projective modules over irrational rotation $\mathrm{C}^{*}$ algebras, Proc. London Math. Soc. (3), 47, (1983), pp. 285-302. MR 85g:46085 
[18] Rieffel, M. A., The homotopy groups of the unitary groups of non-commutative tori, $J$. Operator Theory, 17, (1987), pp. 237-254. MR 88f:22018

[19] Rørdam, M., On the structure of simple $\mathrm{C}^{*}$-algebras tensored with a UHF-algebra, J. Funct. Anal., 100, (1991), pp. 1-17. MR 92m:46091

[20] Villadsen, J., Simple C*-algebras with perforation, J. Funct. Anal., 154, (1998), pp. 110-116. CMP 98:11

Department of Mathematics, Aarhus University, 8000 Arhus C, Denmark

E-mail address: jsv@imf.au.dk

Current address: Institut for Matematik og Datalogi, Odense Universitet, Campusvej 55, 5230

Odense M, Denmark 